\title{
Prognostic Role of Human Equilibrative Transporter 1 (hENT1) in Patients With Resected Gastric Cancer
}

DANIELE SANTINI,' BRUNO VINCENZI,' MARIA ELISABETTA FRATTO,' GIUSEPPE PERRONE, RAYMOND LAI, ${ }^{3}$ VINCENZO CATALANO, ${ }^{4}$ CAROL CASS, ${ }^{3}$ PIER ADELCHI RUFFINI, ${ }^{5}$ CHIARA SPOTO,' PIETRO MURETTO, ${ }^{6}$ SERGIO RIZZO, ${ }^{7}$ ANDREA ONETTI MUDA, ${ }^{2}$ JOHN R. MACKEY, ${ }^{3}$ ANTONIO RUSSO, ${ }^{7 *}$ GIUSEPPE TONINI,' AND FRANCESCO GRAZIANO ${ }^{4}$ 'Medical Oncology, University Campus Bio-Medico, Rome, Italy

${ }^{2}$ Surgical Pathology, University Campus Bio-Medico, Rome, Italy

${ }^{3}$ Cross Cancer Institute and Department of Oncology, University of Alberta, Edmonton, Alberta, Canada

${ }^{4}$ Department of Medical Oncology, Azienda Ospedaliera “Ospedale San Salvatore”, Pesaro, Italy

${ }^{5}$ Divisione Oncologia Medica Falck, Ospedale Niguarda Ca' Granda, Milano, Italy

${ }^{6}$ Institute of Histopathology, Azienda Ospedaliera “Ospedale San Salvatore", Pesaro, Italy

${ }^{7}$ Department of Surgical and Oncological Sciences, Università degli Studi di Palermo, Palermo, Italy

Nucleoside transporter proteins are specialized proteins that mediate the transport of nucleosides and nucleoside analog drugs across the plasma membrane. The human equilibrative nucleoside transporter I (hENTI) is a member of these proteins and mediates cellular entry of gemcitabine, cytarabine, and fludarabine. The hENTI expression has been demonstrated to be related with prognosis and activity of gemcitabine-based therapy in breast, ampullary, lung, and pancreatic cancer. We investigated the immunohistochemical expression of hENT in tumor samples from III patients with resected gastric adenocarcinoma, correlating these data with clinical parameters and disease outcomes. None of the patients received chemotherapy or radiation therapy before or after surgery as a part of an adjuvant or neoadjuvant program. On univariate survival analysis, the hENTI expression was associated with overall survival (OS) and disease free survival (DFS). Specifically, those patients with overexpression of hENTI showed a shorter OS $(P=0.021)$ and a shorter DFS $(P=0.033)$. Considering only the node positive patients, higher hENT levels were associated with significantly shorter median DFS (2I.7 months; $95 \%$ Cl I I.I-32.4) compared with patients with low expression of hENTI. The hENTI expression was defined, in the lymph-node positive patients, as an independent prognostic factor $(P=0.019)$. Furthermore, considering only patients with diffuse or mixed tumors and lymph-node positive, the expression of hENTI was strongly related with DFS and OS. Immunohistochemistry for the hENTI protein carries prognostic information in patients with resected gastric cancer and holds promise as a predictive factor in chemotherapy decisions. J. Cell. Physiol. 223: 384-388, 20I0. (C) 2010 Wiley-Liss, Inc.

Gastric cancer is an aggressive disease. The prognosis of patients remains poor even after radical surgery, because of the unpredictability of the disease and the lack of effective complementary treatments (Ono, 2006). Many molecular prognostic factors based on the biological characteristic of gastric cancer have been proposed in the last few years, but studies based on larger and more homogeneous series of patients are necessary before any of these biological marker can be used in the routine clinical setting for the identification of patients at higher risk of recurrence that could benefit more from adjuvant chemotherapy (Nitti et al., 2008). DNA-ploidy heterogeneity in gastric cancers is also under evaluation as prognostic factor in gastric cancer. To date there is no generally accepted adjuvant chemotherapy for patients with radically resected gastric cancer (Di Costanzo et al., 2008). In advanced gastric cancer the combination between gemcitabine and fluoropyrimidine has been tested, in consideration of the properties of gemcitabine that has shown to enhances the anti-tumor activity of 5-FU.

Nucleoside transporter proteins are specialized proteins that mediate the transport of nucleosides and nucleoside analog drugs across the plasma membrane. This step is necessary for the pharmacological action of these drugs (Molina-Arcas et al.,
2008). The human equilibrative nucleoside transporter I (hENTI), the most abundant and widely distributed plasma membrane nucleoside transporter in human cells, is a member of these proteins and mediates cellular entry of gemcitabine, cytarabine, and fludarabine (Giovannetti et al., 2006b; Zhang et al., 2007). In fact deficiency in hENTI confers resistance to toxicity and efficacy of these drugs in many in vitro models of hematologic (Tallman, 2005; Cai et al., 2008; Lai et al., 2008) and solid tumors (Mackey et al., 1998; Seve et al., 2005; Cano-Soldado et al., 2008). Kinetic studies of human cell lines with defined nucleoside transporter processes have demonstrated that gemcitabine intracellular uptake is mediated

*Correspondence to: Antonio Russo, Section of Medical Oncology, Department of Surgical and Oncological Sciences, School of Medicine, University of Palermo, Via del Vespro 129, 90127 Palermo, Italy. E-mail: lab-oncobiologia@usa.net

Received 8 September 2009; Accepted I December 2009

Published online in Wiley InterScience (www.interscience.wiley.com.), 15 January 2010. DOI: $10.1002 /$ jcp.22045 
greatly by hENTI (Mackey et al., 1998, 1999). It has been demonstrated that ENTI-deficient cells are resistant to cytotoxic nucleosides in vitro (Gati et al., 1998). With the advent of molecular and immunologic probes for nucleoside transporter proteins, the differential expression of nucleoside transporters in normal and tumor tissue has been studied (Pennycooke et al., 200I; Dabbagh et al., 2003).

The abundance and distribution of the hENTI protein can be evaluated using immunohistochemistry and has been assessed in a number of malignant and benign tissues (Mackey et al., 2002; Chow et al., 2005). Recent data indicate that a high expression of $h E N T I$ is a poor prognostic factor in patients with metastatic and resected pancreatic cancer, even if, on multivariate analysis, it is associated with a longer survival, disease-free survival and time to disease progression in patients treated with gemcitabine (Giovannetti et al., 2006a). The identification of high levels of $h E N T I$ represents a positive predictive factor of response to gemcitabine not only in patients with advanced pancreatic cancer (Spratlin et al., 2004), but also in patients with resected disease (Farrell et al., 2007) and with metastatic lung disease (Seve et al., 2005). Recently, our group has demonstrated that hENTI expression is a molecular prognostic marker for patients with resected ampullary cancer, identifying a patient population with poor prognosis that could benefit from nucleoside analog treatment (Santini et al., 2008). On the basis of these findings, we studied the expression of hENT in tumor samples from I I I patients with resected gastric adenocarcinoma, correlating these data with clinical parameters and disease outcomes.

\section{Materials and Methods}

\section{Clinical data and tumor sample acquisition}

This retrospective study targeted a high-risk population of stage II-III gastric cancer patients. A consecutive series of patients from the referral population of a regionally based cancer service were considered. Tissues were collected from patients undergoing gastric surgical resection with curative intent surgery at the Azienda Ospedale San Salvatore (Pesaro, Italy). Only patients without known residual disease were analyzed. One hundred eleven patients with stage II-III disease, which was treated with R0 surgery and DI-D2 lymphoadenectomy ( $>10$ retrieved lymph nodes) (McShane et al., 2005) were included. Data on clinical variables, including sex, age, preoperative assessment of disease status, and type of operative procedure were gathered retrospectively from patient records. A minimum of 3-year followup was required. Follow-up procedures consisted of interim history, physical examination, hematologic evaluation, carcinoembryonic antigen levels, gastrointestinal cancer antigen (GICA), and diagnostic imaging (chest $X$-ray and abdominal ultrasonography) every 4 months in the first year and every 6 months thereafter. Patients underwent upper endoscopy 6 months after surgery and every 12 months thereafter. Whole-body computed tomography was performed for corroborative evidence of relapse. The recurrences of gastric adenocarcinoma had to be confirmed by cytology, biopsy, or surgery. Pathological findings (tumor size, spread, and lymph-node status) were obtained from the pathologists' original reports. Tumor-node-metastasis status (TNM) classification was reassessed using the 1997 revision of the AJCC manual. None of the patients received chemotherapy or radiation therapy before or after surgery as a part of an adjuvant or neoadjuvant program. Survival was determined from the date of initial surgery to the date of death or last contact. Follow-up data were available for all included patients. The study was conducted in a blinded fashion so that patients' outcomes were unknown to investigators performing immunohistochemistry analyses. The study was carried out with approval of the relevant local institutional research boards.

\section{Tissue preparation and immunostaining}

Representative tumor blocks were sectioned at 3- $\mu \mathrm{m}$ thickness for immunohistochemical studies. The sections were deparaffinized with three immersions in xylene baths ( 10 min each) followed by serial washes in graded alcohol from $100 \%$ to $50 \%$. After rinsing in water, slides were placed in $250 \mathrm{ml}$ of high $\mathrm{pH} \mathrm{I} \times$ DAKO target antigen retrieval solution and microwaved in TT-mega Milestone (ESBE Scientific, Markham, Ontario, Canada) under controlled temperature and high pressure for $10 \mathrm{~min}$ at $100^{\circ} \mathrm{C}$. After cooling in water for $6 \mathrm{~min}$, slides were rinsed with water and peroxidase blocked in $3 \%$ hydrogen peroxide solution with methanol for $10 \mathrm{~min}$ then washed in running water for $10 \mathrm{~min}$. Phosphatebuffered saline (PBS) ( $\mathrm{pH} \mathrm{7.2)}$ was used for rinsing before incubation in a humidified chamber overnight at $4^{\circ} \mathrm{C}$ with appropriate dilutions of anti-hENTI mouse mAb developed and characterized as described previously [23]. The sections were then rinsed with PBS, immersed in buffer for $5 \mathrm{~min}$, and incubated with goat anti-mouse dextran conjugate (DAKO Envision) for $30 \mathrm{~min}$ followed by soaking in PBS. DAKO diaminbenzidine liquid chromogen was placed on the samples for $5 \mathrm{~min}$ and rinsed, after which, the slides were soaked in $1 \% \mathrm{CuSO}_{4}$ for another $5 \mathrm{~min}$. Subsequently, the sections were rinsed, counterstained with hematoxylin, dehydrated through graded alcohol and xylene, and finally coverslipped. Negative controls were provided by omitting the primary antibodies. Positive internal controls were provided by evaluation of tissue lymphocytes [2I] and endothelial cells [24] as previously demonstrated. These internal controls were then used as internal reference for the evaluation of relative intensities of stain. In particular, endothelial cell staining was observed and assigned a score of $2+$ and the staining of invasive adenocarcinoma cells was graded as follow: absent (0), definite positive but less intense than internal control tissue $(I+)$, positive-like internal control tissue $(2+)$, and positive, more intense than internal control tissue $(3+)$. Samples with regions of varying staining intensities of hENTI were scored and the percentages of each staining intensity were recorded. Finally, tumors with an intensity staining of $2+$ and $3+$ in more than $50 \%$ of the tumor cells were considered as strong expression of hENTI.

Immunostaining was assessed by two independent pathologists blinded to clinical characteristics and outcomes. In case of different assessment for single case, a third pathologist scored the sample in a blinded way.

\section{Statistical analysis}

A univariate survival analysis for each prognostic variable on overall survival (OS) and disease free survival (DFS) was estimated as by the Kaplan-Meier method (Kaplan and Meier, 1958). The terminal event was death, attributable to cancer or non-cancer causes for OS and clinical detectable progression of disease according to RECIST criteria for DFS. The statistical significance of the differences in survival distribution among the prognostic groups was evaluated by the log-rank test (Peto et al., 1977). The Cox proportional hazards model was applied to the multivariate survival analysis (Cox, 1972). The prognostic variables for OS and DFS included grading, $\mathrm{T}$ and $\mathrm{N}$ factors, stage and hENTI expression values $<0.05$ were regarded as statistically significant in two-tailed tests. SPSS software (Version I3.00, SPSS, Chicago, IL) was used for statistical analysis.

\section{Results \\ Patient characteristics}

The main clinicopathological features of the III studied patients are summarized in Table I. Median age was 72 years (range 36-88); 53 were males and 58 females. Seventy-nine (7I.2\%) patients had locoregional lymph-node metastasis. Forty-seven (42.3\%) patients had stage II disease, whereas $57.7 \%$ had stage III. In this study, 48 patients (43.2\%) died with 
TABLE I. Patients' characteristics

\begin{tabular}{lc}
\hline Total number & $1 \mathrm{II}$ \\
Age (years) & \\
Median (minimum-maximum) & $72(36-88)$ \\
Gender: number (\%) & \\
Male & $53(47.8 \%)$ \\
Female & $58(52.2 \%)$ \\
Gastrectomy: number (\%) & \\
Total & $79(71.2 \%)$ \\
Partial & $32(28.8 \%)$ \\
Histology: number (\%) & \\
Diffuse & $27(24.3 \%)$ \\
Intestinal & $68(61.3 \%)$ \\
Mixed & $15(14.4 \%)$ \\
Stage: number (\%) & \\
2 & $47(42.3 \%)$ \\
3 & $64(57.7 \%)$ \\
Grading: number (\%) & $6(5.4 \%)$ \\
Well differentiated & $59(53.1 \%)$ \\
Moderately differentiated & $46(41.5 \%)$ \\
Poorly differentiated & \\
Pathologic tumor status & $21(18.9 \%)$ \\
PT2 & $88(79.2 \%)$ \\
PT3 & $2(1.9 \%)$ \\
PT4 & $79(71.2 \%)$ \\
Lymph-node status: number (\%) & $32(28.8 \%)$ \\
Positive & \\
Negative & $9.3(0-87.5)$ \\
Percentage of positive nodes & \\
Median (minimum-maximum) & $58(52.2 \%)$ \\
Relapse: number (\%) & $53(47.8 \%)$ \\
No & $63(56.7 \%)$ \\
Yes & $48(43.3 \%)$ \\
Death: number (\%) & \\
No & \\
Yes & \\
\hline &
\end{tabular}

positive cytological or histological confirmation of recurrent disease. All 48 relapsed patients underwent 5-fluorouracil/ cisplatin-based palliative chemotherapy. Median follow-up after surgery was 5.5 years (range 3-8.5 years). After this follow-up, 58 patients $(52.5 \%)$ are still alive without evidence of recurrence, 5 patients $(4.5 \%)$ are alive with recurrence of disease, and 48 patients (43\%) are dead for cancer recurrence.

\section{Clinicopathological characteristics and patients' outcome}

In our cohort of patients a significantly improved OS was observed in patients with low-grade $(P=0.002)$, low $T$ $(P=0.04 \mathrm{I})$, $\mathrm{N}$ negative tumors $(P=0.020)$, and low tumor stage $(P=0.001)$ on univariate analysis. A significant statistical correlation was also found between grading $(P=0.002)$, $\mathrm{N}$ status $(P=0.022)$, tumor stage $(P=0.001)$, and DFS on univariate analysis (Kaplan-Meyer curves not shown).

\section{hENTI staining}

The immunohistochemical hENTI staining was present within tissue lymphocytes and endothelial cell, as previously reported, and were used as internal positive controls. The localization of the hENTI immunostaining was predominantly membrane, although occasional cytoplasmic staining was seen. The normal gastric mucosa and the peritumoral stroma cells were otherwise negative. Of the I I0 tumor specimens, 50 (45.4\%) specimens had high hENTI immunostaining (intensity scores $>50 \%$ ), while 60 (54.5\%) samples were classified as low hENTI immunostaining (intensity scores $\leq 50 \%$ ). Of 68 intestinal type gastric cancers, 30 (44.I\%) were classified as high hENTI immunostaining patients. Of 27 diffuse type cancers, 7 (25.9\%) were classified as high hENTI immunostaining patients.

\section{Correlation between clinical outcomes and hENTI staining}

To establish the prognostic role of hENTI, patients were stratified in positive (hENT-high expression) versus negative (hENT-low expression). On univariate survival analysis, the hENTI expression was associated with OS and DFS.

Specifically, those patients with overexpression of hENTI showed a shorter OS $(P=0.02 \mathrm{I})$ and a shorter DFS $(P=0.033$; Fig. I).

\section{hENTI staining and survival: Subgroup analysis}

After having established that $\mathrm{hENTI}$ is a negative prognostic factor in all patients, we evaluated if the expression of hENT have a role also in the subsets of patients with poor prognosis. Of the III patients, 79 (7I.2\%) of the patients were lymph-node positive. Considering only these patients, we demonstrated on univariate survival analysis that higher hENT levels were associated with significantly shorter median DFS (21.7 months; 95\% Cl I I.I-32.4, Fig. 2) compared with patients with low expression of hENTI (median DFS not reached). These results were confirmed on multivariate analysis, in fact the hENTI expression was defined as an independent prognostic factor ( $P=0.019$, Table 2$)$. In lymph-node positive patients, a correlation between hENTI levels and OS was also
A

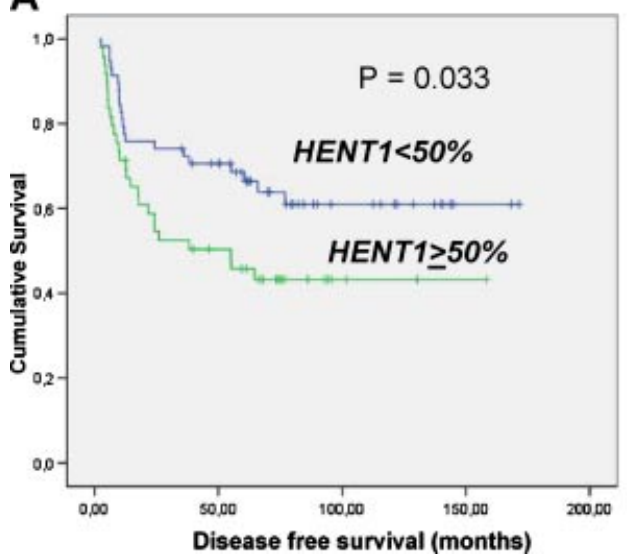

B

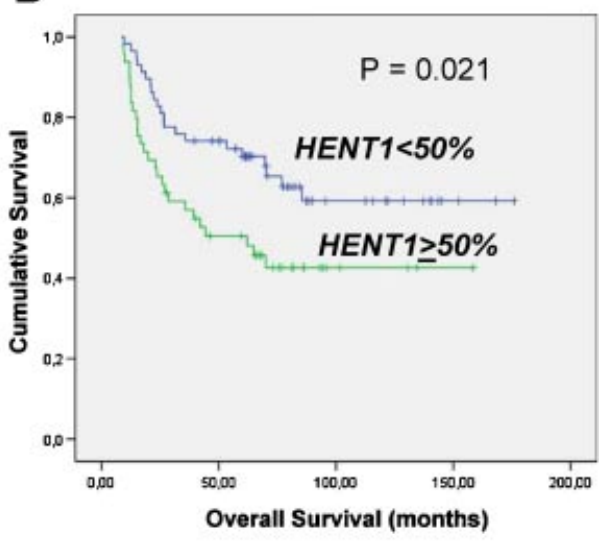

Fig. I. Correlation between hENT expression and DFS (A) and OS (B). [Color figure can be viewed in the online issue, which is available at www.interscience.wiley.com.] 


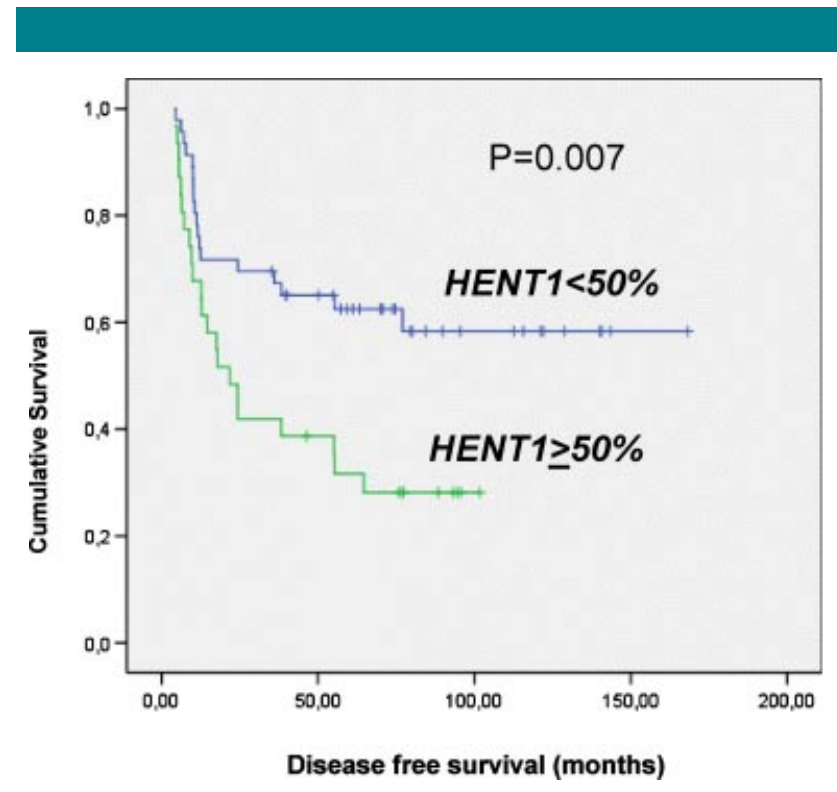

Fig. 2. Correlation between hENT expression and DFS in lymphnode positive patients: univariate analysis. [Color figure can be viewed in the online issue, which is available at www.interscience. wiley.com.]

demonstrated on univariate analysis. In fact hENTI-positive patients had a shorter median OS (31.533 months; $95 \% \mathrm{Cl} 10$, 295-II.355, Fig. 3) in comparison to hENT-negative patients (median OS not reached). Our findings from the multivariate analysis demonstrated that the hENT-I expression was also a strong independent predictor of OS ( $P=0.017$, Table 3$)$.

\section{Conclusions}

There are few substantial data reporting significant biological prognostic markers for gastric cancer patients. The influence of pathologic (tumor stage, lymph-node involvement, status of resection margins) and surgical (aggressive surgical approach on lymph nodes versus limited resection) factors is clear (Kirkwood et al., 1997). There is, in radically resected gastric cancer, substantial interest in identifying and validating molecular markers to select patients with a high likelihood of benefiting from specific chemotherapy regimens. Gemcitabine, a nucleoside chemotherapy drug used in many solid tumors, requires the nucleoside transporter protein hENTI to efficiently enter cells (Mackey et al., 1998), and hENTI deficiency confers resistance to gemcitabine in vitro (Mackey et al., 1999). Moreover, hENTI plays an important role in 5-FU resistance in vitro (Tsujie et al., 2007). Recently, the immunohistochemical assessment of the nucleoside

TABLE 2. Multivariate analysis of DFS in lymph-node positive patients

\begin{tabular}{|c|c|c|c|}
\hline & Relative risk & 95\% Confidential interval & $P$ \\
\hline \multicolumn{4}{|l|}{ DFS } \\
\hline \multicolumn{4}{|l|}{ Grading } \\
\hline $1-2$ & I & - & 0.027 \\
\hline 3 & 1.946 & $1.21-2.99$ & \\
\hline \multicolumn{4}{|l|}{ Histology } \\
\hline Intestinal & 1 & - & 0.445 \\
\hline Diffuse-mixed & 1.210 & $0.83-1.86$ & \\
\hline \multicolumn{4}{|l|}{ HENT-I } \\
\hline 0 & 1 & - & 0.019 \\
\hline I & 2.125 & $1.18-3.78$ & \\
\hline
\end{tabular}

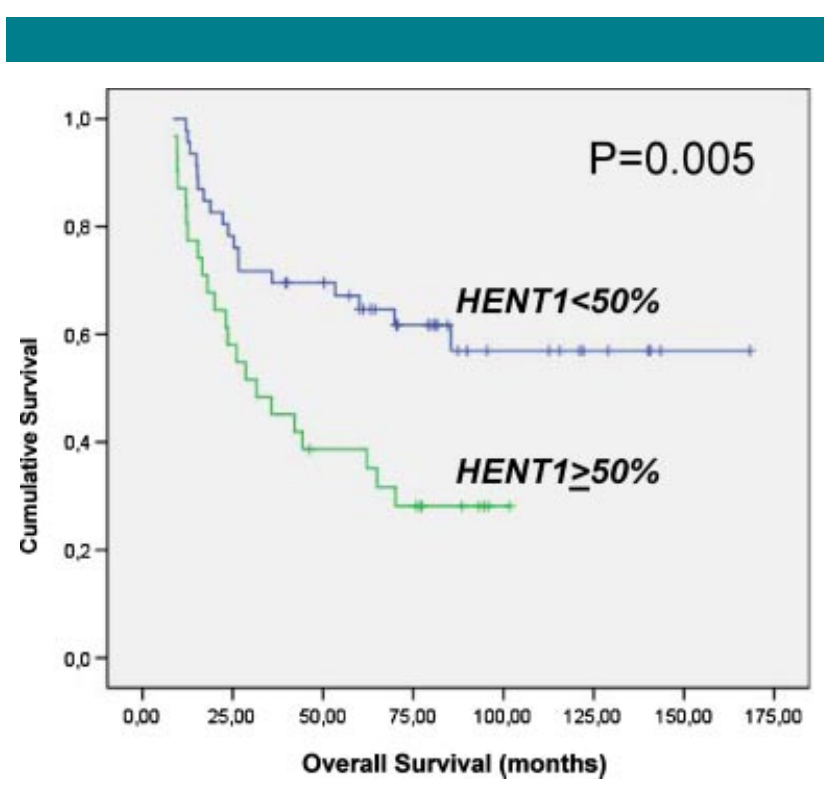

Fig. 3. Correlation between hENT expression and OS in lymphnode positive patients: univariate analysis. [Color figure can be viewed in the online issue, which is available at www.interscience. wiley.com.]

transporter protein hENTI has shown both prognostic and predictive value in the setting of adenocarcinoma of the pancreas and of lung cancer treated with the nucleoside chemotherapy drug, gemcitabine (Mackey et al., 2002; Chow et al., 2005; Seve et al., 2005). For these reasons, we characterized the pattern of expression to explore the possible prognostic significance of hENTI expression in a homogeneous cohort of patients with radically resected gastric cancer. Similar to the previous description of hENTI in pancreatic carcinoma (Chow et al., 2005) immunohistochemical identification of the hENTI protein in gastric cancer tissue revealed two patterns of staining, either (a) positive staining of adenocarcinoma cells or (b) regions lacking $\mathrm{hENTI}$ in a proportion of adenocarcinoma cells.

Internal controls for this assessment were provided by positively staining endothelial cells and lymphocytes. In this cohort of patients we demonstrated that $45.4 \%$ of specimens had high hENTI immunostaining (intensity scores $2-3+$ in $>50 \%$ ), while $54.5 \%$ of samples possessed a low or absent hENTI immunostaining (intensity scores $2-3+$ in $\leq 50 \%$ ). Our hypothesis is that at least one half of patients may have a higher probability to benefit from gemcitabine-based and fluoropyrimidine-based chemotherapy. However, further studies with gemcitabine/fluoropyrimidines-treated gastric carcinoma assessed for hENTI immunostaining will be required

TABLE 3. Multivariate analysis of OS in lymph-node positive patients

\begin{tabular}{lccc}
\hline & Relative risk & $95 \%$ Confidential interval & $P$ \\
\hline $\begin{array}{c}\text { OS } \\
\text { Grading } \\
\text { I-2 } \\
3\end{array}$ & & & \\
$\begin{array}{c}\text { Histology } \\
\text { Intestinal } \\
\text { Diffuse-mixed }\end{array}$ & 1 & - & 0.027 \\
$\begin{array}{c}\text { HENT-I } \\
0\end{array}$ & 1.944 & $1.20-3.28$ & \\
I & 1 & - & 0.445 \\
\hline
\end{tabular}


to evaluate this hypothesis. This study clearly showed that high immunohistochemical staining for the hENTI protein is significantly correlated with poor prognosis, in term of OS and DFS, in patients with radically resected gastric cancer. Moreover, considering only positive node patients, higher hENT levels were associated with significantly shorter median DFS and OS. These results were confirmed on multivariate analysis, in fact the hENTI expression was defined as an independent prognostic factor for DFS and OS (respectively, $P=0.019$ and 0.017 ). In the node positive subset of patients hENTI expression could represent also a predictive factor for response to gemcitabine/fluoropyrimidines-based chemotherapy in adjuvant setting. These findings warrant the consideration of prospective trials of gemcitabine/ fluoropyrimidines based chemotherapy in resected gastric carcinoma, with tissue assessment to define the relationship between hENTI immunohistochemical staining and clinical outcomes. Furthermore, gemcitabine and fluorouracil or capecitabine may be of particular benefit in those patients with metastatic or recurrent disease expressing high levels of hENT I in tumor samples. In conclusion, immunohistochemistry for the $\mathrm{hENTI}$ protein carries prognostic information in patients with resected gastric cancer and holds promise as a predictive factor to assist in chemotherapy decisions. Moreover, these findings will be validated using also other modalities.

\section{Literature Cited}

Cai J, Damaraju VL, Groulx N, Mowles D, Peng Y, Robins MJ, Cass CE, Gros P. 2008. Two distinct molecular mechanisms underlying cytarabine resistance in human leukemic cells. Cancer Res 68:2349-2357.

Cano-Soldado P, Molina-Arcas M, Alguero B, Larrayoz I, Lostao MP, Grandas A, Casado FJ, Pastor-Anglada M. 2008. Compensatory effects of the human nucleoside transporters on the response to nucleoside-derived drugs in breast cancer MCF7 cells. Biochem Pharmaco 75:639-648.

Chow L, Lai R, Dabbagh L, Belch A, Young JD, Cass CE, Mackey JR. 2005. Analysis of human equilibrative nucleoside transporter I (hENTI) protein in non-Hodgkin's lymphoma by immunohistochemistry. Mod Pathol 18:558-564.

Cox DR. 1972. Regression models and life tables. J R Stat Soc 34:187-220.

Dabbagh L, Coupland RW, Cass CE, Mackey R. 2003. Immunohistochemical variation of human equilibrative nucleoside transporter I protein in primary breast cancers. Clin Cancer Res 9:3213-3214.

Di Costanzo F, Gasperoni S, Manzione L, Bisagni G, Labianca R, Bravi S, Cortesi E, Carlini P, Bracci R, Tomao S, Messerini L, Arcangeli A, Torri V, Bilancia D, Floriani I, Tonato M, Dinota A, Strafiuso G, Corgna E, Porrozzi S, Boni C, Rondini E, Giunta A, Monzio Compagnoni B, Biagioni F, Cesari M, Fornarini G, Nelli F, Carboni M, Cognetti F, Enzo MR Piga A, Romiti A, Olivetti A, Masoni L, De Stefanis M, Dalla Mola A, Camera S, Recchia F, De Filippis S, Scipioni L, Zironi S, Luppi G, Italia M, Banducci S, Pisani Leretti A, Massidda B, lonta MT, Nicolosi A, Canaletti R, Biscottini B, Grigniani F, Di Costanzo F, Rovei R, Croce E, Carroccio R, Gilli G, Cavalli C, Olgiati A, Pandolfi U, Rossetti R, Natalini G, Foa P, Oldan S, Bruno L, Cascinu S, Catalano G, Catalano V, Lungarotti F, Farris A, Sarobba MG Trignano M, Muscogiuri A, Francavilla F, Figoli F, Leoni M, Papiani G, Orselli G, Antimi M, Bellini V, Cabassi A, Contu A, Pazzola A, Frignano M, Lastraioli E, Saggese M, Bianchini D, Antonuzzo L, Mela M, Camisa R. 2008. Adjuvant chemotherapy in completely resected gastric cancer: A randomized phase III trial conducted by GOIRC. J Natl Cancer Inst 100:388-398.
Farrell J, Garcia M, Lai R, Ammar A, Regine W, Abrams R, Bowen Benson A, Macdonald], Cass $\mathrm{CE}$, Elsaleh H, Mackey J. 2007. Human ENTI is predictive of response to gemcitabine treatment in patients with pancreatic cancer: Results from the RTOG 9704 prospective randomized trial. Pancreas 35:40I-402.

Gati WP, Paterson ARP, Belch AR, Chlumecky V, Larratt LM, Mant MJ, Turner AR. 1998. Es nucleoside transporter content of acute leukemia cells: Role in cell sensitivity to cytarabine. Leuk Lymphoma 32:45-54

Giovannetti E, Del Tacca M, Mey V, Funel N, Nannizzi S, Ricci S, Orlandini C, Boggi U, Campani D, Del Chiaro M, lannopollo M, Bevilacqua G, Mosca F, Danesi R. 2006a. Transcription analysis of human equilibrative nucleoside transporter-I predicts survival in pancreas cancer patients treated with gemcitabine. Cancer Res 66:3928-3935.

Giovannetti E, Mey V, Nannizzi S, Pasqualetti G, Del Tacca M, Danesi R. 2006b.

Pharmacogenetics of anticancer drug sensitivity in pancreatic cancer. Mol Cancer Ther 5:1387-1395.

Kaplan E, Meier P. 1958. Non parametric estimation from incomplete observations. J Am Stat Assoc 53:457-48I.

Kirkwood KS, Khitin LM, Barwick KW. 1997. Prognostic indicators for cancer. Gastric cancer. Surg Oncol Clin N Am 6:495-5I4.

Lai R, Bartlett NL, Mackey JR, Jung SH, Johnson JL, Cook JR, Jones D, Cass CE, Young JD, Said J Cheson B, Hsi ED. 2008. High expression of nucleoside transporter protein hENTI in Reed-Sternberg cells is associated with treatment failure in relapsed/refractory Hodgkin lymphoma patients treated with gemcitabine, vinorelbine and liposomal doxorubicin-A CALGB 59804 correlative study. Leuk Lymphoma 49:1202-1205.

Mackey JR, Mani RS, Selner M, Mowles D, Young JD, Belt JA, Crawford CR, Cass CE. 1998 Functional nucleoside transporters are required for gemcitabine influx and manifestation of toxicity in cancer cell lines. Cancer Res 58:4349-4357.

Mackey JR, Yao SY, Smith KM, Karpinski E, Baldwin SA, Cass CE, Young JD. 1999. Gemcitabine transport in xenopus oocytes expressing recombinant plasma membrane mammalian nucleoside transporters. J Natl Cancer Inst 91:1876-1881.

Mackey JR, Jennings LL, Clarke ML, Santos CL, Dabbagh L, Vsianska M, Koski SL, Coupland RW, Baldwin SA, Young JD, Cass CE. 2002. Immunohistochemical variation of human equilibrative nucleoside transporter I protein in primary breast cancers. Clin Cancer Res 8:110-116.

McShane LM, Altman DG, Sauerbrei W, Taube SE, Gion M, Clark GM. 2005. Reporting recommendations for tumor marker prognostic studies. I Clin Oncol 23:9067-9072

Molina-Arcas M, Trigueros-Motos L, Casado FJ, Pastor-Anglada M. 2008. Physiological and pharmacological roles of nucleoside transporter proteins. Nucleosides Nucleotides Nucleic Acids 27:769-778.

Nitti D, Mocellin S, Marchet A, Pilati P, Lise M. 2008. Recent advances in conventional and molecular prognostic factors for gastric carcinoma. Surg Oncol Clin N Am 17:467-483, vii. $\mathrm{OnoH}$. 2006. Early gastric cancer: Diagnosis, pathology, treatment techniques and treatment outcomes. Eur J Gastroenterol Hepatol 18:863-866.

Pennycooke M, Chaudrey N, Shuralyova I, Zhang Y, Coe IR. 200I. Differential expression of human nucleoside transporters in normal and tumor tissue. Biochem Biophys Res Commun 280:95I-959.

Peto R, Pike MC, Armitage P, Breslow NE, Cox DR, Howard SV, Mantel N, McPherson K Peto J, Smith PG. 1977. Design and analysis of randomized clinical trials requiring prolonged observation of each patient. II. Analysis and examples. Br J Cancer 35: I-39.

Santini D, Perrone G, Vincenzi B, Lai R, Cass C, Alloni R, Rabitti C, Antinori A, Vecchio F, Morini S, Magistrelli P, Coppola R, Mackey JR, Tonini G. 2008. Human equilibrative nucleoside transporter I (hENTI) protein is associated with short survival in resected ampullary cancer. Ann Oncol 19:724-728.

Seve P, Mackey JR, Isaac S, Tredan O, Souquet PJ, Perol M, Cass C, Dumontet C. 2005. cN-II expression predicts survival in patients receiving gemcitabine for advanced non-small cell lung cancer. Lung Cancer 49:363-370.

Spratlin J, Sangha R, Glubrecht D, Dabbagh L, Young JD, Dumontet C, Cass C, Lai R, Mackey IR. 2004. The absence of human equilibrative nucleoside transporter I is associated with reduced survival in patients with gemcitabine-treated pancreas adenocarcinoma. Clin Cancer Res 10:6956-6961.

Tallman MS. 2005. New strategies for the treatment of acute myeloid leukemia including antibodies and other novel agents. Hematology Am Soc Hematol Educ Book I:143-150.

Tsujie M, Nakamori S, Nakahira S, Takahashi Y, Hayashi N, Okami J, Nagano H, Dono K. Umeshita K, Sakon M, Monden M. 2007. Human equilibrative nucleoside transporter I, as a predictor of 5 -fluorouracil resistance in human pancreatic cancer. Anticancer Res 27:224I-2249.

Zhang J, Visser F, King KM, Baldwin SA, Young JD, Cass CE. 2007. The role of nucleoside transporters in cancer chemotherapy with nucleoside drugs. Cancer Metastasis Rev 26:85-110. 